\title{
Self-care assessment as an indicator for clinical supervision in nursing
}

\author{
Avaliação do autocuidado como indicador para supervisão clínica em enfermagem
}

Sílvia Marlene Monteiro Teixeira ${ }^{1}$, António Luís Rodrigues Faria de Carvalho ${ }^{2}$, Sandra Sílvia da Silva Monteiro Santos Cruz ${ }^{2}$

Objective: to evaluate the needs of clinical supervision for nurses to assess the degree of dependence on selfcare and planning of nursing interventions. Methods: analytical study, cross-cutting nature, collecting data from a sample of 110 patients. Results: it was shown the differences in the identification of the degree of dependence between registers and experts, as well as the selection of operations for each self-care and failures to the original assessment of the filling level (no evaluation self-care/no identification of the degree of dependence). Conclusion: there were gaps in the nursing process; they have proposed strategies such as clinical supervision sessions, training, case studies, protocols and guidance documents, to be included in a clinical supervision in nursing model.

Descriptors: Nursing; Self Care; Quality of Health Care; Preceptorship.

Objetivo: avaliar as necessidades dos enfermeiros em supervisão clínica para avaliar o grau de dependência no autocuidado e planear intervenções de enfermagem. Métodos: estudo analítico, recolhendo dados de uma amostra de 110 doentes. Resultados: evidenciaram-se diferenças na identificação dos graus de dependência entre registos e especialistas, bem como na seleção das intervenções para cada autocuidado e falhas ao nível do preenchimento da avaliação inicial (não avaliação dos autocuidados/descuro na identificação dos graus de dependência). Conclusão: identificaram-se lacunas no processo de enfermagem; propuseram-se estratégias como sessões de supervisão clínica, formação, estudo de casos, protocolos e guias de orientação, a serem incluídas em um modelo de supervisão clínica em enfermagem.

Descritores: Enfermagem; Autocuidado; Qualidade da Assistência à Saúde; Preceptoria.

\footnotetext{
${ }^{1}$ Obra Diocesana de Promoção Social. Porto, Portugal

${ }^{2}$ Escola Superior de Enfermagem do Porto. Porto, Portugal. 


\section{Introduction}

The Clinical Supervision appears closely linked to nursing the nurturing quality of care and its effects in this area are current affairs and timely ${ }^{(1)}$. A growing number of studies aimed at the exploration and evaluation of the effectiveness of clinical supervision have been recently published in the nursing field, but its heart has rarely been the evolution of the patient or the quality of care $^{(2)}$. Currently, the experience and research in clinical supervision in nursing in Portugal are expanding ${ }^{(3)}$, and the areas identified as essential for potential health gains should be prioritized and developed intervention models ${ }^{(4)}$.

Ensuring the safety of patients and professionals and improving the quality of health services are priority objectives of any health system ${ }^{(5)}$.

With the problem under study, "the assessment of self-care as an indicator for clinical supervision in nursing" is intended to contribute to the construction of a clinical supervision model in nursing to ensure good clinical practice of higher quality and safer, specifically under the self-care, the development of nurses' intervention skills in this area.

Self-care is a focus of major nursing care, defined by the Order of Nurses as an indicator of the quality of health care ${ }^{(6)}$. Its promotion is considered one of the goals of the nurses. Thus, self-care has been selected as an area sensitive to the influence of Clinical Supervision in focus in this research, contributing to enhance the reflection and awareness of professionals on the practices in use, thus participating more effectively, to change them.

The quality of care has become an area of interest and concern for healthcare organizations and their professionals, particularly nurses so that the committee formation of the Nurses Order proposes it as a priority area for research in nursing in Portugal. Nurses are key professionals in the responsibility to participate in quality improvement activities; in its field of action is self-care that is an indicator of a professional exercise that aims for excellence.
It is undeniable that the improvement of nursing care requires a reflective practice that can be operationalized through clinical supervision in nursing ${ }^{(7)}$, an important instrument for the development of their quality ${ }^{(8)}$.

Supervision Clinical Nursing is defined "as a formal monitoring process [continued] of professional practice, which aims to promote making independent decisions, valuing the person's protection and safety of care through processes of reflection and analysis clinical practice" ${ }^{\prime(9: 5)}$. It is a relational process aimed at the support and monitoring of supervised while nursing professional, by a supervisor, focusing on the development of knowledge and reflective skills of the first on the practice of nursing care to ensure improved quality and excellence. Clinical supervision allows for increased job satisfaction and well-being feeling, preventing burnout ${ }^{(10)}$ and contributing to the effectiveness and efficiency of nurses ${ }^{(8)}$. For such benefits happen, it has to be operationalized by applying a model, elaborating individual plans of the orientation of supervision process enabling the provision of safe nursing and quality care ${ }^{(8)}$, based on scientific evidence, which is a tool for the development of nursing as a profession and even as a science.

Once complexity characterizes the nurse, by the link to a multitude of patients, each with different requirements, and also by the existence of diverse needs of the professionals the range of models is varied.

Self-care concept with increasing importance in healthcare, much by the progressive aging of the population and the prevalence of chronic diseases is defined as an "Activity Executed by the Own: dealing with the need to keep operating and handling the basic and intimating the individual needs and activities of daily living according to the International Classification for Nursing Practice ${ }^{(11: 41)}$. It involves actions such as bathing, undressing or dressing, eating, organizing, taking care of personal hygiene, self-esteem, using the toilet, moving up, turning around and using the wheelchair ${ }^{(11)}$. 
This article aimed to evaluate the needs of clinical supervision for nurses to assess the level of dependence on self-care plan and nursing interventions.

\section{Methods}

Conducted within the research project "Clinical Supervision for Safety and Quality of Care", this study is analytical.

Framed in the first phase of the research of this project, the aim was to make a diagnosis of the clinical context face areas sensitive to nursing care later to build and implement a clinical supervision model in nursing able to respond to identified needs for intervention, contributing to improving the quality and safety of the implemented practices.

The research was developed in a hospital in the Northern region of Portugal. The study population was composed of patients admitted to the medical service of this hospital. The sample of 110 elements, was obtained through a non-probabilistic sampling, using accidental sampling or convenience and included all patients admitted to the medical service A, except those to whom it was not possible to gather data transfer service or short-term hospitalization (24-48 hours).

Data collection occurred in a single moment by applying two instruments: the first self-care assessment tool consisted of three parts. The first part relates to the characterization of the patient; the second part includes the dependency assessment tool self-care developed and validated ${ }^{(12)}$ and finally the selection of nursing interventions according to the dependency degree identified. It is emphasized that this instrument was completed by rehabilitation nursing specialists. The second assessment tool selfcare, directed to the analysis of nursing records, also comprises three parts identical to the previous instrument and completed by the investigator during the document analysis, based on records in Support System Nursing Practice, Nursing process, and the initial assessment.
The use of these two data collection instruments aimed to provide a concordance between the data obtained from the records (performed by general nurses) and the instrument filled by specialist nurses in rehabilitation.

The statistical treatment of the data was performed using the Statistical Package for Social Sciences software (version 19.0), having performed procedures of descriptive and inductive statistics to draw conclusions from the information obtained.

To characterize the sample, we performed a descriptive analysis of the variables. To verify differences between nominal variables, we used the Wilcoxon test (Z).

For all tests and analyses performed, statistical significance was accepted at a value of $\mathrm{p}<0.05$.

The study complied with the formal requirements contained in the national and international standards of regulatory research involving human beings.

\section{Results}

The sample consisted of 42 women and 68 men, aged between 25 and 95 years old. The average age of participants was 73.14 years old ( $\mathrm{SD}=12.86)$. In both genders, the predominant age group was 65-79 years old. Of the elements under study, 61 (55.5\%) were dependent at home in some self-care $(69.0 \%$ of the female sample and $47.0 \%$ of the male sample), and the age group that recorded more cases of independence were between 41-64 years old and higher dependence from 80 years old.

There was a variety of diagnostic groups, and three had a higher frequency of cases: diseases and disorders of the respiratory system in 47 elements $(42.7 \%)$, then the diseases and disorders of the circulatory system in 30 cases $(27.3 \%)$ and diseases and disorders of the kidney and urinary tract in 12 cases $(10.9 \%)$.

On admission of a patient, an initial assessment is carried out to identifying needs and further 
diagnosis. In $79.9 \%(n=78)$ was a reference to selfcare in this primary evaluation, and in all these cases the given self-care is self-care hygiene. Of them, only 18 cases there is the degree of dependence, but in six this identification was made in "motor activity" and not in self-care; in one case it was not mentioned any self-care, but it is referred to a degree of dependence.

Through the different self-care and the degree of dependence, none of the self-care is the full correspondence of the evaluation made by experts about the degree of dependence of the different self-care and nursing records that reflect the care of patients.

By applying the Wilcoxon test ( $\mathrm{Z}$ ), there were the results shown in Table 1 the tested hypothesis confirmed except self-care variable Transfer.

Table 1 - Differences between the degrees of dependency

\begin{tabular}{lcc}
\hline Self-care & $\mathbf{Z}^{*}$ & $\mathbf{p}$ \\
\hline Take a bath & $-5,317$ & $<0,001$ \\
Dressing / undressing & $-8,265$ & $<0,001$ \\
Feeding & $-3,016$ & 0,003 \\
Personal arrangement & $-7,916$ & $<0,001$ \\
Take care of personal hygiene & $-5,392$ & $<0,001$ \\
Self-esteem & $-7,972$ & $<0,001$ \\
Use of health & $-4,013$ & $<0,001$ \\
Movign up & $-1,115$ & 0,265 \\
Turn around & $-3,676$ & $<0,001$ \\
Using the wheelchair & $-7,341$ & $<0,001$ \\
\hline Wilcoxon Test & &
\end{tabular}

After the behavioral study sample on the degree of dependency, it was important to know whether the planned interventions were adequate to the degree of dependence identified. Thus, there was a sample distribution analysis for total correspondence partially, does not correspond or not to apply the selected interventions and dependency levels, for each self-care, both for data collected those skilled nurses and the registers of which resulted in that for the specialist nurses, there is no self-care interventions that do not correspond to the degree of addiction and all self-care there are cases of partial match. In the records, it was found that there are cases of mismatch and, as in specialist nurses, the Move Wheelchair variable is self-care with lower total correspondence.

Specifically, for interventions related to equipment and adaptive strategies, it was found that in specialist nurses in all self-care interventions were selected in the context of teaching, instructing and training, while the records it was found that in any self-care such intervention was selected.

There are statistically significant differences ( $p$ $<0.05$ ) between the interventions selected by experts and records.

\section{Discussion}

The study identified the needs of nurses to provide nursing care safer and with higher quality in the self-care. Although this is a study in the context of a health facility, the study explains a method to identify the aspects to be included in clinical supervision model to develop strategies to promote the personal and professional development of nurses ${ }^{(13)}$. The method used to identify issues to be addressed in clinical supervision can be replicated in all situations and with all indicators.

The results show the need for strategies that contribute to a better assessment of self-care and a better planning of nursing interventions.

In $79.9 \%$ of cases, self-care was mentioned in the initial evaluation although only on the hygiene. Although the percentage referred to be high, the remaining cases are causing concern since, with the initial assessment a tool to agglomerate all knowledge relating to the patient upon admission, which subsequently is useful for the nursing team Plan better and more uniformly throughout the care relocation, it would expect that it would contain all the relevant information. The initial assessment should be as complete as possible; being self-care nursing a focus of extreme importance, it must not be omitted. When mentioned self-care, the degree of dependence was 
often overlooked (only 16.4\% - 18 cases - was found). Of these 18 cases, there were cases where the degree of dependence was not mentioned in item mentioned for self-care but in on the motor activity.

Regarding the degree of dependence on selfcare, in their records, the focus "muddle" was used indiscriminately, being placed in the specifications when the diagnostic construct, it is reported that the use of a walker, cane, crutches or wheelchair. The construction of a diagnosis should presuppose the use of focus that specifically describe the situation. It is considered that with a specific focus on the use of the wheelchair - "Moving in a wheelchair," it must be used for proper effect.

In no part of the sample using the focus "Dressing or undressing" but was visible but the "SelfCare Clothing". This may have happened because there is some confusion of concepts between this two focus (the correct identification would be the "Dress up or undressing") or by the fact that the parameterization, the "Dressing or undressing" be two separate focus that does not justify the use of a wrong focus. In this sense, it is necessary to clarify the concepts used to provide nursing care appropriate to the actual needs of patients.

In any self-care was the complete correspondence of the degree of dependence between the specialist nurses and records. Statistically, significant differences were found between the degree of dependence records and specialist nurses, except in the variable transfer.

Intervention "Evaluate ..." selected in all cases by specialist nurses was not in the records of any element of the sample not be parameterized in computerized nursing system, which does not mean that it was not implemented in practice since, to check the maintenance or not the degree of dependence of a self-care, you must do it.

There were identified statistically significant differences in operations between the two data sources in all self-care. The replacements have been the most recorded and those in which manifests the greatest correspondence between specialist nurses and records. We must give importance to this information by calling attention to the fact that commonly the workload that nurses have with the patient the diagnosis that identified and interventions prescribed/performed. It is necessary to sensitize professionals to critically reflect the planning of their care and guide them to the real needs of patients.

Data collected from records stands out without interventions diagnoses were identified, and associated interventions selected not associated with any diagnosis. It reinforces that was not detected a single intervention related to teaching, instruct and train, which raises the possibility of being made but not recorded.

From the above, it was possible to identify gaps that relate to the nursing process. The same should be targeted for intervention under the clinical supervision of nursing since said process is essential for the care system. The decision-making influences the design and delivery of care, both in the way the nurses carry out their activities and interventions, and in their choices ${ }^{(12)}$. Supervision Clinical Nursing emerges as a key tool to give nurses more knowledge and skills ${ }^{(13)}$.

It is important that the dependence profile (type and grade) were evaluated using the appropriate tools recording the evolution of the patient's condition that, when measurable, could translate into a quality process indicator. The quality of information, particularly registered, must be systematically improved through the architecture of computer systems supporting the practice and training of professionals ${ }^{(4)}$.

It is necessary to develop appropriate models of intervention in these cases mirrored in the implementation of a clinical supervision model in nursing, using the research to test the effectiveness and efficiency of care and thus find the best answers to the needs of populations ${ }^{(1-4,7-8,10,14)}$.

From the above, and focusing on practices aimed at self-care, they are proposed as improvement strategies and developing skills of nurses, to be effected 
by the implementation of a Clinical Supervision Model in Nursing:

Training ${ }^{(4)}$ : the nursing team with the selfcare approach (domains, styles, influencing factors, the nurse's role). It should work the critical and reflective spirit, useful to improve the diagnosis and intervention process;

Case studies: training ${ }^{(4)}$ of mental abilities and thinking about building a thought process nursing diagnosis, as a team;

Development of action protocols: the existence of a protocol directed to the nurses on self-care approach would allow standardization of practices contributing to the process efficiency and continuity of care. This protocol would have to include steps to implement to reach the correct diagnosis identity and define to be built and designed by the team, for each possible diagnosis, and according to the specifics of each patient, the most appropriate and effective interventions;

Preparation of guidance documents ${ }^{(15)}$ : a decision-making process and implementation of interventions nurses should have incorporated the most current knowledge (on practical self-care) and more tailored to your work environment, the result of nursing research;

Group supervision sessions: allow the elements of a team effort and reflects agreeing among themselves on issues that concern us all, and individual supervision sessions: to address unmet needs for group supervision.

\section{Conclusion}

The study demonstrated the need to identify the needs of supervision on nurses to provide the safest and highest quality care.

There were gaps in the nursing process identified; They have proposed strategies such as clinical supervision sessions, training, case studies, protocols and guidance documents, to be included in a clinical supervision in nursing model.

It is concluded that for a correct intervention by nurses, it is necessary that all the diagnosis and care planning process is thought in detail, considering the characteristics of each patient, but also professionals and resources within the context of clinical practice. It is called to the awareness of nursing professionals for greater investment in the development of these skills so that in this way, the care provided are of an effective quality. In this context, the Supervisory Clinical Nursing plays a key role in response to identified needs of both patients and professionals. But this is only achievable if it is sustained in-depth studies of practices in the specific context of work.

Continuous improvement of nurses' performance should be promoted, focusing on research in priority areas such as self-care.

\section{Collaborations}

Teixeira SMM contributed in data collection, presentation, analysis and discussion of the results and also in the design and writing of the article. Carvalho ALRF and Cruz SSSMS contributed to the design and orientation of the project, article writing, critical review of its content and final approval of the version to be published.

\section{References}

1. Hyrkäs K, Lethi K. Continuous quality improvement through team supervision supported by continuous self-monitoring of work and systematic patient feedback. J Nurs Manag. 2013; (11):17788.

2. Dilworth S, Higgins I, Parker V, Kelly B, Turner J. Examining clinical supervision as a mechanism for changes in practice: a research protocol. J Adv Nurs. 2014; 70(2):421-30.

3. Cruz S. Clinical supervision in nursing: effective pathway to quality. Procedia Soc Behav Sci. 2011; (29):286-91. 
4. Ministério da Saúde (PT). Alto comissariado para a saúde - PNS 2011-2016: estratégias para a saúde - objetivos para o sistema de saúde: obter ganhos em saúde (Versão Discussão). Lisboa: Ministério da Saúde; 2011.

5. World Health Organization (WHO). Standards for Health Promotion in Hospitals: development of indicators for a self-assessment tool. Copenhaga: WHO; 2004.

6. Ordem dos Enfermeiros (PT). Conselho de Enfermagem. Padrões de qualidade dos cuidados de enfermagem - Enquadramento conceptual; Enunciados descritivos. Lisboa: Ordem dos Enfermeiros; 2012.

7. Cruz S, Carvalho L. Portuguese version of the Manchester Clinical Supervision Scale ${ }^{\odot}$ : the translation and validation process. J Educ Sci Psychol. 2012; II-LXIV(1):123-31.

8. Koivu A, Saarinen P I, Hyrkas K. Who benefits from clinical supervision and how? The association between clinical supervision and the work-related well-being of female hospital nurses. J Clin Nurs. 2012; 21:2567-78.

9. Ordem dos Enfermeiros (PT). Caderno temático: modelo de desenvolvimento profissional fundamentos, processos e instrumentos para a operacionalização do sistema de certificação de competências. Lisboa: Ordem dos Enfermeiros; 2010.
10. Wallbank S. Maintaining professional resilience through group restorative supervision. Comm Pract. 2013; 86(8):26-8.

11. International Council of Nurses. Classificação Internacional para a Prática de Enfermagem (CIPE): versão 2.0. Lisboa: Ordem dos Enfermeiros; 2011.

12. Duque H. O doente dependente no autocuidado: estudo sobre a avaliação e acção profissional dos enfermeiros. Porto: ESEP; 2009.

13. Cruz S, Carvalho AL, Sousa P. Clinical supervision: priority strategy to a better health. Procedia Soc Behav Sci. 2014; 112:97-101.

14. Cruz S, Carvalho A, Sousa P. Clinical supervision and emotional intelligence capabilities: Excellence in clinical practice. Procedia Soc Behav Sci. 2015; 171:153-7.

15. Ordem dos Enfermeiros (PT). Cuidados à pessoa com alterações de mobilidade - posicionamentos, transferências e treino de deambulação: guia orientador de boa prática. Lisboa: Ordem dos Enfermeiros; 2013. 